\title{
Prospectiva y escenarios probabilísticos de la Nacionalidad Tsáchila
}

\section{Prospective and probabilistic scenarios of Nationality Tsachila}

*David A. Arias Chávez ${ }^{1}$, Johana Abril Ortega ${ }^{1}$, Jenny Núñez Moreno ${ }^{1}$, Ángel Zapata Barros ${ }^{1}$

${ }^{1}$ Pontificia Universidad Católica del Ecuador Sede Santo Domingo (PUCESD), Escuela de Ciencias Administrativas y Contables, Vía a Chone y San Cristóbal, Código Postal 230203, Santo Domingo de los Tsáchilas, Ecuador

*acda@pucesd.edu.ec; aоje@pucesd.edu.ec; nmjp@pucesd.edu.ec, zbae@pucesd.edu.ec

Fecha de recepción: 20/06/2017 Fecha de aceptación: 10/07/2017

Publicado: 24/12/2017

\section{Resumen}

La metodología de prospectiva estratégica es de origen francés y avalada por la UNESCO; su propósito es dilucidar el futuro con escenarios de probabilidad relativa de ocurrencia, esta herramienta es considerada para prescribir orientaciones estratégicas a la Nacionalidad Tsáchila y la convergencia del Plan de Vida de la Etnia, acorde a los lineamientos del Plan Nacional del Buen Vivir en el objetivo 5. Este método contiene una etapa de reflexión colectiva, posición de actores y juego de hipótesis probabilizadas, que se esgrimen en tres softwares. El primer sistema nombrado MICMAC, permite la identificación de variables esenciales y proporciona resultados visuales en planos de influencias y dependencias, para verificar las hipótesis elaboradas por el grupo de expertos sobre la explicación del fenómeno en estudio. Los Tsáchilas, se encuentran en un proceso de preservación ancestral, es comprensible que stakeholders influyan directa e indirectamente sobre ellos; identificados estos grupos de interés se adhieren al segundo sistema denominado MACTOR, que a través de gráficos de convergencia y divergencia permiten visualizar la posición de los actores. Con las derivaciones obtenidas en los métodos de MICMAC y MACTOR, se aplica el software SMIC PROB EXPERT, sistema que catalizó la identificación de escenarios futuros de 
ocurrencia que podría enfrentar la Etnia, y se contrastó con la visión del Plan de Vida de la

Nacionalidad para justificar si converge con el escenario de mayor probabilidad relativa.

Palabras clave: grupo étnico, investigación social, patrimonio cultural inmaterial, planificación del desarrollo, prospectiva.

\begin{abstract}
The methodology of strategic foresight has a French origin and it's endorsed by UNESCO; its purpose is to elucidate the future with relative probability of occurrence scenarios, this tool is considered to prescribe strategic guidelines to the Tsáchila Nationality and the convergence of the Ethnia Plan of Life, according to the guidelines of the National Plan for Good Living on the objective $5^{1}$. This method contains a collective thinking stage, players position and probability hypothesis game, which are hold on three softwares. The first system called MICMAC allows the identification of essential variables and provides visual results in levels of influences and dependencies, to verify the hypothesis made by the expert group on the explanation of the phenomenon under study. Tsáchilas are in an ancestral preservation process, it is understandable that stakeholders directly and indirectly affect them; identified these interest groups, they adhere to the second system called MACTOR, that through convergence and divergence charts allows to visualize the position of the actors. With the leads obtained in MICMAC and MACTOR methods, it's applied the SMIC PROB EXPERT software, system that identified future scenarios of occurrence that the ethnicity could face, and it was contrasted with the vision of Nationality Plan of Life to justify if converges with the most likely relative scenario.
\end{abstract}

Keywords: ethnic group, social investigation, intangible cultural heritage, development planning, prospective. 


\section{Introducción}

La prospectiva es la forma de analizar el futuro de un sistema, se caracteriza por una visión holística en la que los actores y las variables son la clave en la construcción de un escenario persistentemente accesible a cambios que depende de la acción del hombre, y por la cual esta se convierte en una herramienta fundamental de planeación, que dilucida lo que ocurrirá y orienta las gestiones humanas para la realización de lo esperado.

Esta herramienta proyectiva aporta una reflexión futurible para la conservación de tendencias ancestrales y el compromiso social de fortalecer la identidad nacional y de analizar las posibles condiciones del futuro de una de la Etnias más importantes del Ecuador, partiendo desde un patrón histórico. Antecedentes sobre la nacionalidad Tsáchila datan del año de 1542, según Canelos (2004).

En el Atlas de las lenguas del mundo de la UNESCO, en la diversidad lingüística, el idioma del pueblo Tsáchila denominado Tsa fiki es catalogado en peligro, por el número de locutores que han desaparecido en un $92.67 \%$ y existentes entre 2000 a 2200 comuneros; el nivel de seguimiento y de las tendencias que efectúa esta organización ha establecido que este acontecimiento se debe a la transmisión intergeneracional de la lengua, en la cual los niños ya no la aprenden en sus familias como lengua materna.

Con el estudio prospectivo, se identificarán desde un punto de vista retrospectivo y actual variables, grupos sociales que son gestores del desarrollo del cambio y posibles escenarios que enfrentará la etnia, los cuales coadyuvaran a verificar si la visión del Plan de Vida de la Nacionalidad Tsáchila, converge con el escenario de mayor grado de probabilidad relativa, denominado tendencial. 
La metodología recurrida en este análisis prospectivo, para discernir y construir un futuro se basa en herramientas técnicas y tecnológicas, implementando tres sistemas que se correlacionan; MICMAC que consiente a evidenciar las relaciones entre variables en función del criterio de influencia y dependencia, categorizando a ocho tipos de variables estratégicas, para establecer las denominadas claves; que constituirán los elementos de mayor influencia en las comunas que componen la Nacionalidad.

El MACTOR, software que estima la correlación de fuerzas que existen entre los grupos interesados y las convergencias y divergencias con respecto a determinados objetivos asociados, identificando los cuatro tipos de actores que son clasificados en el plano de influencias y dependencias, para verificar su alto o bajo nivel de incidencia en el macro y micro ambiente de la comunidad; y finalmente el SMIC PRO EXPERT que en función de una combinación binaria de cada escenario se logrará el grado de cumplimiento de los retos estratégicos que enfrentará en el largo plazo la Nacionalidad Tsáchila, bajo los criterios de las probabilidades simples y condicionales positivas y negativas.

\section{Materiales y Métodos}

La reflexión prospectiva que se presenta en este trabajo no pretende predecir el futuro extrapolando el pasado en base al comportamiento estadístico de un conjunto de variables cuantitativas como sugiere el enfoque Forward que se menciona en el trabajo de Vázquez y Ortegón 2006, es más bien una aproximación al futuro de la nacionalidad Tsáchila a partir de una imagen objetivo (escenarios). Este análisis se propone conocer que le espera a esta etnia indígena en los próximos años (futuros posibles) para evaluar en qué medida las acciones y decisiones presentes están conduciendo al futuro deseado (escenario deseable), por lo tanto se aborda a la investigación 
prospectiva bajo un enfoque Backward, que supuso entender el futuro de la Nacionalidad Tsáchila ( sistema) como una construcción colectiva condicionada por variables (internas y externas) y actores que al combinarse pueden generar no solo un futuro sino varios.

El menú diario de la prospectiva es considerar las rupturas posibles o los puntos de bifurcación. Identificar la gama de futuros posibles por el método de escenarios permite definir las rupturas potenciales, los caminos que conducen a ellos y las consecuencias que entrañan. Los parámetros de esos cambios constituyen variables clave para el análisis de la prospectiva (Godet y Durance, 2011, p. 32).

El análisis del futuro desde el futuro (Backward) sigue el proceso metodológico planteado por Cely, A. V. (1999) que sugiere tres etapas de desarrollo prospectivo que inician con un análisis estructural que estableció las variables clave del sistema (Nacionalidad Tsáchila), está lectura permitió definir cuál es el escenario deseable desde la perspectiva de esta nacionalidad objeto de análisis y las principales variables y actores que lo condicionan. "La normatividad significa tener en cuenta la propuesta de futuros deseables que manifiesta una comunidad científica, empresarial gubernamental o social" (Vásquez y Ortegón, 2006, p. 161).

En una segunda fase se evaluó el papel de los actores en el desarrollo del sistema, este análisis permitió obtener información sobre cómo se encuentra actualmente este grupo social; es decir, la realidad en donde los actores sociales toman decisiones que condicionan el futuro. En un tercer momento se presenta la realidad futura en forma de escenarios y se contrastan las dos realidades planteadas (actual y futura) con la finalidad de construir un marco de orientación global que permita conocer hacia dónde se dirige este pueblo, para esta etapa fue necesario utilizar la herramienta 
MICMAC (Matriz de Impactos Cruzados Multiplicación Aplicada a una Clasificación) que modelizó el problema a abordar.

La observación de los cambios sociales consiste en realizar un rastreo de las tendencias, los eventos y los propósitos de los actores sociales en el tiempo. Significa preguntarse de dónde venimos, dónde estamos y para dónde podemos ir. Consiste en verificar mediante hechos, datos e interpretaciones, de dónde procede el sistema social, cuál es su origen, su estado actual y su posible evolución (Godet y Durance, 2011, p. 162).

\section{Análisis Estructural}

El análisis estructural sigue tres pasos secuenciales que se inician con un listado de las variables que caracterizan y condicionan la realidad (presente y futura) de los Tsáchilas, en esta etapa inicial del estudio se utilizaron 43 variables definidas a partir de un sondeo a expertos, a continuación se determinó la relación que existe entre las variables seleccionadas con un criterio cualitativo $(0=$ nulo, 1 = débil, 2 = mediana, 3 = fuerte, $\mathrm{P}$ = potencial) esta relación permite al programa construir una matriz de impactos cruzados (relación múltiple y dinámica de la variables) de la cual se extraen las variables clave. Las variables clave son clasificadas a partir de la influencia que estas ejercen sobre el sistema, esta influencia se valora con el criterio de motricidad-dependencia que sirve para tipificar las relaciones directas, indirectas y potenciales entre variables. Son variables clave las que ejercen mucha influencia sobre otras variables y no son muy influenciadas por éstas, y son también clave las variables que influencian mucho sobre otras variables pero también están condicionadas por éstas.

\section{Análisis del Juego de Actores}


El análisis de involucrados siguió tres de los cinco pasos mencionados en el trabajo de Vázquez y Ortegón (2006), identificando 31 involucrados en base a un consenso dentro del equipo de investigación, luego se clasificó a los involucrados en base al criterio de Cely, A. V. (1999), como involucrados poder, producción, saber y comunidad. Involucrados poder, el estado y sus instituciones; producción, las empresas u otros sectores productivos; saber, son instituciones educativas; y comunidad, en general. Finalmente se determinó las relaciones de poder que vinculan a los involucrados entre sí y con el sistema a través del método MACTOR.

\section{Elaboración de Escenarios}

Para la determinación de los escenarios se utilizó el software SMIC PROB EXPERT que a partir de la información entregada por el programa MICMAC y el método MACTOR y siguiendo un proceso de dos etapas (formulación de hipótesis y elección de expertos y probabilización de escenarios) se construyen escenarios posibles a los que el sistema analizado podría enfrentarse. "Los escenarios constituyen una guía indispensable para orientar las decisiones estratégicas. El método de los escenarios ayuda a determinar la estrategia más adecuada para la realización del proyecto deseado, pues permite poner de relieve los mayores retos organizativos" (Godet y Durance, 2011, p. 56).

\section{Resultados y Discusión}

\section{Proceso de Identificación y Categorización de las Variables}

Esta etapa constituye la base fundamental en la estructuración del sistema estudiado, para la identificación de las variables es necesario realizar previamente un diagnóstico exhaustivo a nivel interno, el cual se desarrolló mediante un trabajo de campo en las siete comunas que conforman la Etnia, las cuales se ubican de forma dispersa en una extensión de 10.050,1 hectáreas $(100.50$ 
kilómetros cuadrados), éste procedimiento permite establecer potencialidades y limitaciones. Por otra parte la evaluación externa contempla la observación de los factores de mayor incidencia del macro entorno, relacionados con aspectos tecnológicos, políticos, legales, económicos y ambientales.

Puede existir una cantidad significativa de variables; pero es preciso focalizarse en los criterios vinculados con los puntos de apalancamiento e implicaciones del ámbito de la Nacionalidad Tsáchila. Éste es un mecanismo semicuantitativo de selección, que correlaciona elementos del estudio situacional, en una matriz que prioriza puntos inherentes de conversión de factores, favorables y desfavorables, sean estos del entorno o de la concepción central del grupo étnico.

Cada análisis prospectivo, mantiene una categorización diferente de acuerdo a la naturaleza de lo que se está examinado, este estudio en particular, se enfoca a 9 criterios comunes; que fueron establecidos con entrevistas a expertos a nivel nacional, en este caso a la máxima autoridad del grupo étnico y como contexto internacional, a los agregados culturales de las embajadas de la República Popular de China, Perú, Egipto y Colombia.

Una vez realizada la identificación de los factores claves, se procede a preparar la información para el análisis estructural del sistema estudiado, con este propósito se elabora un inventario de variables mediante un trabajo colectivo a través una minería de datos.

La lista de componentes, constituyen elementos homogéneos, que adicionalmente al ser identificados, deben describirse, codificarse y categorizarse (Godet, 1993); posteriormente son ingresados al programa informático de LIPSOR denominado MIC MAC ( Matriz de Impactos Cruzados, Multiplicación Aplicada a una Clasificación) con el objeto de realizar una transición de una metodología semicuantitativa a un esquema cuantitativo de procesamiento de la información. 
En este estudio en particular se ha identificado 43 variables, las cuales serán parte de un proceso sistémico que permitirá evidenciar las relaciones entre variables en función del criterio de influencia y dependencia. Subsiguientemente se utiliza una tabla de dos entradas, (Godet, 2004) denominada como "matriz de análisis estructural" y en base a la formula (n $x \mathrm{n}-1)$, se establece que se deben realizar 1806 preguntas de interrelación entre variables, y en función a éste proceso de interrogación exhaustivo, se califica su incidencia en el sistema, considerando un rango de 0 a 3.

El resultado de este mecanismo se lo evidencia gráficamente en el plano de influencias y dependencias directas, Figura 1, donde se puede verificar que las 43 variables se encuentran

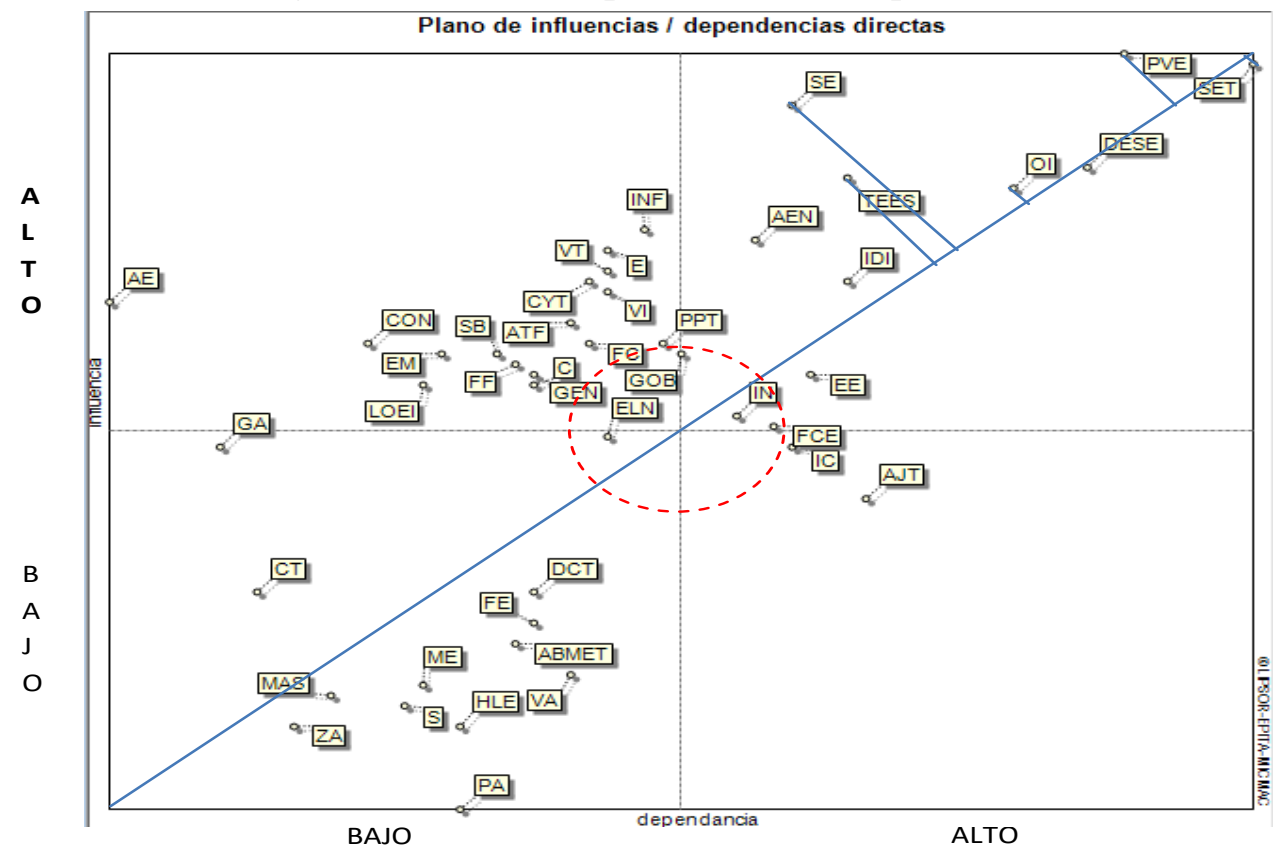

Figura 1. Plano de influencias y dependencias directas

Fuente: software LIPSOR MICMAC

dispersas en los 4 cuadrantes y según su nivel alto o bajo de influencia y dependencia, se realiza su respectiva categorización.

Las Variables Objetivo son componentes que se encuentran ubicados en el nivel medio de influencia y alto de dependencia, estas variables constituyen la base de las ideas de innovación estratégica, las que se mencionan seguidamente: AEN (Ambiente Étnico Natural), IDI (Idioma), 
EE (Equidad de la Etnia).

Por otra parte las Variables secundarias se localizan sobre la diagonal en la zona inferior izquierda, se identifican por ser más influyentes que dependientes; dentro de este contexto se identifican las siguientes: GA (Granjas Avícolas), CT (Circunscripción Territorial). En relación a las Variables dependientes o de Resultado, estos componentes se los encuentra en la zona inferior derecha, en donde el nivel de influencia es bajo y la dependencia es alta, en este sector se localiza a la IC (Identidad Cultural) y AJT (Administración de la Justicia Tsáchila).

También constan las Variables Autónomas, éstas se colocan en la zona inferior izquierda, mantienen un bajo nivel de influencia y dependencia, dado que no constituyen parte determinante para el sistema en el largo plazo y se identifican en esta ubicación el DCT (Derecho Consuetudinario Tsáchila), VA (Vestimenta y Accesorios), FE (Folklore de la Etnia), MAS (Medicina Ancestral y Shamanismo), ME (Modelo Educativo), ABMET (Acervo Bibliográfico y Material Educativo Tsafique), ZA (Zonas Arqueológicas), S (Salud), HLE (Historias y Leyendas de la Etnia) y PA (Producción Agrícola).

En otra zona las Variables del Entorno, constituyen elementos que condicionan el sistema dado su mediano grado de influencia y baja dependencia, aquí se localizan las AE (Actividades de las empresas), CON (Contaminación), LOEI (Ley Orgánica de Educación Intercultural), EM (Explotación del Material), SB (Servicios Básicos), C (Conectividad), ATF (Asistencia Técnica y Financiera), FC (Fiestas y Celebraciones), FF (Flora y Fauna) y GEN (Género). Las Variables determinantes e influyentes, se establecen dentro del plano como de alta influencia poca dependencia, en este segmento se localizan, INF (Infraestructura), E (Educación), VT (Vialidad y transporte), CYT (Ciencia y Tecnología) y VI (Vivienda e Infraestructura). Por otra parte las 
Variables reguladoras se agrupan en el círculo de la intersección de los cuatro cuadrantes, en esta posición se encuentra la IN (Integración de la Nacionalidad), GOB (Gobernabilidad), ELN (Estatuto y Legislación de la Nacionalidad), PPT (Propiedad y Posesión de las Tierras) y FCE (formación y Capacitación de la Etnia).

Finalmente las Variables claves o estratégicas, constituyen elementos que se encuentran en el cuadrante superior derecho, en la zona de enlace, donde existe un alto grado de influencia y dependencia, su importancia es relevante, dado que justifican la formulación de los eventos o hipótesis dentro del estudio prospectivo, en este sector se ubica la SET (Sustentabilidad de la Etnia), PVE (Plan de vida de la Etnia), DESE (Defensa Étnica / Supervivencia Étnica), OI (Organización Indígena), TEES (Turismo Étnico - Ecológico Sustentable) y SE (Seguridad Jurídica).

\section{Juego de Actores - MACTOR}

El análisis estratégico del juego de actores constituye una de las etapas valiosas de la prospectiva, Godet (2009) menciona que a través del método de análisis denominado MACTOR, busca estimar la correlación de fuerzas que existen entre los grupos interesados y estudiar sus convergencias y divergencias con respecto a determinados retos y objetivos asociados. Los juegos de alianzas y de conflictos potenciales entre las partes interesadas en el método MACTOR contribuyen en la formulación de cuestiones clave de la prospectiva, y en las recomendaciones estratégicas sobre las posibilidades de evolución de relaciones entre los concernidos, emergencia, desaparición y cambios de rol de los mismos. En la teoría de juegos, los actores son los grupos que persiguen proyectos diferentes, condicionan la evolución del sistema estudiado, e inquieren en la resolución de conflictos existentes. El grupo de expertos, para la construcción del cuadro de estrategia de las partes comprometidas, se interesó por aquellos que dominan las variables claves,

resultantes del análisis estructural compuesto por el árbol de competencias, estereotipos y cambios 
Revista Ciencias Sociales y Económicas - UTEQ (2017)

ISSN 2588-0586 IMPRESO; ISSN 2588-0594 ELECTRÓNICO

Volumen 1, Número 2. Semestral (julio-diciembre)

esperados para el futuro de la Etnia, como parte de la fase de reflexión colectiva, procedimiento que determinó 31 actores que influyen en las variables Tabla 1. 


\begin{tabular}{|c|c|c|c|c|}
\hline $\mathbf{N}^{\circ}$ & Actores & Codificación & MMIDI & \\
\hline 1 & Tsáchilas & TSA & 1 & \\
\hline \multirow[t]{2}{*}{2} & Gobierno Central del & GCE & 1.4 & \\
\hline & \multicolumn{4}{|l|}{ Ecuador } \\
\hline 3 & GAD Municipal & GAD M & 1.2 & \\
\hline 4 & GAD Provincial & GADP & 1.2 & \\
\hline 5 & CODENPE & $\mathrm{CDP}$ & 1 & \\
\hline 6 & FENOCIN & FNC & 0.9 & \\
\hline 7 & FEINE & FIE & 0.8 & \\
\hline 8 & CONAIE & $\mathrm{COE}$ & 0.9 & \\
\hline \multirow[t]{2}{*}{9} & Ministerio de Cultura y & $\mathrm{MCP}$ & 1 & \\
\hline & Patrimonio & & & \\
\hline \multirow[t]{2}{*}{10} & Ministerio Coordinador & MCDS & 0.9 & \\
\hline & de Desarrollo Social & & & \\
\hline \multirow[t]{3}{*}{11} & Comisión de los & CDCCI & 0.9 & Enlace \\
\hline & Derechos Colectivos & & & \\
\hline & Comunitarios & & & \\
\hline \multirow[t]{3}{*}{12} & Comisión de la & CBRN & 0.9 & Autónomo \\
\hline & Biodiversidad y Recursos & & & \\
\hline & Naturales & & & \\
\hline \multirow[t]{2}{*}{13} & Asamblea Nacional de la & ANRE & 1.2 & Dominante \\
\hline & República del Ecuador & & & \\
\hline
\end{tabular}




\begin{tabular}{|c|c|c|c|c|}
\hline 14 & Población de Santo & PSDZU & 0.9 & Autónomo \\
\hline & Domingo Zona Urbana & & & \\
\hline 15 & $\begin{array}{l}\text { Tenientes de Gobernación } \\
\text { de Cabildos Comunales }\end{array}$ & TGCC & 0.8 & Dominado \\
\hline 16 & $\begin{array}{l}\text { Consejo del Gobierno } \\
\text { Tsáchila }\end{array}$ & CGT & 1 & Dominado \\
\hline 17 & $\begin{array}{l}\text { Gobernador de la Etnia } \\
\text { Tsáchila }\end{array}$ & GET & 1 & Dominado \\
\hline 18 & $\begin{array}{l}\text { Presidentes de los } \\
\text { cabildos Comunales }\end{array}$ & PCC & 0.8 & Dominado \\
\hline 19 & $\begin{array}{l}\text { Congreso de la } \\
\text { Nacionalidad Tsáchila }\end{array}$ & $\overline{\mathrm{CNT}}$ & 1 & Dominado \\
\hline 20 & $\begin{array}{l}\text { Población de Santo } \\
\text { Domingo Zona Rural }\end{array}$ & PSDR & 0.8 & Autónomo \\
\hline 21 & $\begin{array}{l}\text { Gobernador de la } \\
\text { Provincia }\end{array}$ & GP & 0.9 & Enlace \\
\hline 22 & Ministerio de Finanzas & $\mathrm{MF}$ & 1.1 & Autónomo \\
\hline 23 & Ministerio de Turismo & MT & 1.1 & Enlace \\
\hline 24 & $\begin{array}{l}\text { Población Activa del } \\
\text { Ecuador }\end{array}$ & PAE & 1.3 & Enlace \\
\hline 25 & $\begin{array}{l}\text { Población Inactiva del } \\
\text { Ecuador }\end{array}$ & PIE & 1.1 & Autónomo \\
\hline
\end{tabular}




\begin{tabular}{|c|c|c|c|c|}
\hline 26 & $\begin{array}{l}\text { Población de Santo } \\
\text { Domingo }\end{array}$ & PSD & 1.2 & Enlace \\
\hline 27 & $\begin{array}{l}\text { Secretaría de los Pueblos } \\
\text { Movimientos Sociales } \\
\text { PC. }\end{array}$ & SPPC & 1.1 & Dominante \\
\hline 28 & $\begin{array}{l}\text { Ministerio de Trasporte y } \\
\text { Obras Públicas }\end{array}$ & MTOP & 0.9 & Enlace \\
\hline 29 & $\begin{array}{l}\text { Secretaría Nacional de } \\
\text { Gestión de Riesgos }\end{array}$ & SNGR & 0.9 & Enlace \\
\hline 30 & Ministerio de Salud & MS & 1 & Autónomo \\
\hline 31 & Ministerio de Educación & $\mathrm{ME}$ & 0.8 & Autónomo \\
\hline
\end{tabular}

\section{Fuente: Software Liptor-Epita-MACTOR}

Nota. Los grupos de interés dominado o sumiso, se ubican en el cuadrante inferior derecho, los cuales tienen una alta dependencia y una baja influencia; ubicados en el cuadrante superior derecho, con alta dependencia e influencia los stakeholders de enlace. Los grupos autónomos, se ubican en el cuadrante inferior izquierdo, son aquellos que tienen una baja dependencia y alta influencia; finalmente en la parte superior izquierda los dominantes con baja dependencia y alta influencia.

La metodología Delphi de análisis lógico conduce a conclusiones sobre el futuro, por medio de la deliberación y juicio de los expertos en los diferentes procesos de cambio del sistema en estudio, que se valoró en el cuadro de estrategia de los grupos de interés obtenidos en la fase de reflexión colectiva.

Los stakeholders identificados en el sistema, persiguen proyectos múltiples y variados, encuentros entre sí mismos, en función de sus finalidades, de los propósitos y medios 
de acción que son asociados; lo que permite revelar un cierto número de posturas estratégicas sobre las cuales estos tendrán objetivos convergentes o divergentes. Con la utilización del programa MACTOR se calculó la fuerza de acción de los interesados y el criterio del grado de influencia; (Godet, 2009) lo estable en cinco niveles de relaciones.

El nivel ponderado es suficientemente preciso para estimar de antemano las posibilidades de alianzas o de conflictos y bastante reducido en número para que el análisis resulte fácil, ya que esta etapa condiciona la calidad de relaciones ulteriores.

El software a partir de los cálculos en la matriz de actores por actores (MID), establece la convergencia entre estos, por medio de un gráfico donde se visualiza como aliados al Gobierno Central del Ecuador (GCE), a la Comisión de la Asamblea Nacional del Ecuador (CBRN) y los Tsáchilas (TSA); los que concuerdan sus ideas e intereses para la sustentabilidad de la Etnia; en la divergencias entre actores de orden 3, se refleja que existe una fuerte discrepancia entre la Asamblea Nacional de la República del Ecuador (ANRE), los Tsáchilas (TSA); Ministerio de Transporte y Obras Públicas y Ministerio de Educación (ME). Posteriormente, en la siguiente fase del método MACTOR, considerando las seis variables de enlace propuestas en la primera fase del estudio se precisa un número de seis objetivos a juicio del investigador, Tabla 2.

\section{Retos Estratégicos}




\begin{tabular}{llcc}
\hline $\mathrm{N}^{\circ}$ & \multicolumn{1}{c}{ Título largo } & $\begin{array}{c}\text { Título } \\
\text { corto }\end{array}$ & $\begin{array}{c}\text { 2 } \\
\text { MAO }\end{array}$ \\
\hline 1 & $\begin{array}{l}\text { La organización indígena promueve el fortalecimiento } \\
\text { administrativo de la etnia. }\end{array}$ & LAO & 29 \\
& $\begin{array}{l}\text { El Plan de Vida de la Etnia está fundamentado en el proceso } \\
\text { equilibrado que permite mantener el desarrollo integral. }\end{array}$ & & \\
\hline 3 & $\begin{array}{l}\text { La Sustentabilidad de la etnia está fundamentada en el } \\
\text { proceso equilibrado que permite mantener el desarrollo }\end{array}$ & & \\
& LAP & 39 \\
integral. & & & \\
\hline 4 & $\begin{array}{l}\text { Defensa étnica y /o Supervivencia radica en la integración } \\
\text { de los miembros de la etnia enfocados a objetivos comunes. }\end{array}$ & & 58 \\
\hline 5 & $\begin{array}{l}\text { La Seguridad Jurídica garantiza el derecho consuetudinario } \\
\text { de la nacionalidad }\end{array}$ & LSJ & 38 \\
\hline 6 & $\begin{array}{l}\text { El Turismo étnico - ecológico promueve el progreso cultural } \\
\text { de la nacionalidad }\end{array}$ & ETE & 62 \\
\hline
\end{tabular}

Seguidamente, en la matriz de posiciones de actores por objetivos, con la sumatoria de los valores positivos y negativos de las filas se establecen los stakeholders más identificados con los objetivos, así como los retos estratégicos de mayor aceptación. En la metodología del MACTOR, se visualiza el histograma $2 \mathrm{MAO}$ en el cual se interpreta el compromiso de los grupos de interés en los objetivos 6 y 4 con una puntuación alta del 62 y 58, con una calificación media de 42 y 39 para los desafíos 2 y 3; y una valoración de 38 y 29 para las hipótesis 6 y 1, Tabla 2.

Posteriormente, se analiza la ambivalencia entre las partes interesadas, en los resultados obtenidos no se presenta actores con este tipo de característica, ya que su ponderación 
da como resultado cero. En el histograma MMIDI se puede demostrar que los grupos de interés internos del sistema son aquellos que tienen relaciones de fuerza más débiles del juego de actores, que presentan una fuerza de 0 . La relación de fuerza más favorable la demuestra el Gobierno Central del Ecuador con una ponderación de 1.4, Población Activa del Ecuador con un valor de 1.3, Población de Santo Domingo, Asamblea Nacional de la República del Ecuador, GAD Provincial y GAD Municipal con un valor de 1.2; los stakeholders restantes tienen relaciones de fuerzas intermedias, Tabla 1.

Los stakeholders antes mencionados al pertenecer al grupo de la fuerza mas favorable, permiten su inserción eficaz en el Plan de Vida de la Etnia Tsáchila y la sustentabilidad de la misma, por medio de la organización y el turismo étnico - ecológico, garantizando la seguridad jurídica y el defensa étnica.

\section{Análisis de Convergencia entre la Visión General del Plan de Vida de la Nacionalidad y los Escenarios de Mayor Probabilidad Relativa}

Los Tsáchilas al igual que otros pueblos indígenas del Ecuador han experimentado procesos de aculturación y transculturación derivados de la colonización y expansión del modo de vida occidental, este proceso ha puesto en riesgo la existencia futura de esta sociedad ancestral como lo advierte el atlas de las nacionalidades 2010 en donde se menciona que el Tsa'fiki está en peligro de desaparecer. Frente a esa realidad en el año 2012 fue presentado el plan de vida de la nacionalidad Tsáchila (PVNT) como resultado del consenso dialogal de este pueblo que tiene como prioridad fundamental 
no desaparecer. Sin embargo si se compara el PVNT con el escenario tendencial del estudio se concluye que las prácticas vivenciales actuales no están enfocadas a garantizar la supervivencia colectiva de los Tsáchilas, esto se debe principalmente a que ha existido un deterioro de la actividad económica tradicional (recolección, caza, agricultura, pesca y artesanía) que ha hecho necesario el desarrollo de iniciativas económicas nuevas (turismo) que se plantearon de manera individual (en cada comuna) y no asociativa (cadenas de producción asociadas), está condición promueve la competencia entre comunidades y contradice su filosofía ancestral de vida (Seke Sonano), si estas prácticas se mantienen existe un $34.2 \%$ de probabilidad de que este grupo indígena no alcance el futuro deseado (sobrevivir como pueblo).

La situación actual de la Nacionalidad Tsáchila es la realidad de las minorías excluidas en el Ecuador, es decir tienen problemas de necesidades básicas insatisfechas (educación, salud, vivienda, infraestructura, etc.), dificultades para sostener sus actividades económicas, desigual tenencia y mercantilización de tierras comunales y alta concentración de la PEA en actividades agrícolas, toda esta problemática les categoriza bajo el paradigma de vida occidental como un pueblo no desarrollado, esta marginación más el proceso de aculturación y transculturación han generado entre sus miembros el legítimo anhelo de bienestar, pero como lo refleja el PVNT este anhelo se acerca cada vez más a los paradigmas de vida mestizos (visión occidental de vida) en donde se entiende el bienestar desde una perspectiva economisista-reduccionista. A partir de la premisa anterior y si se compara el PVNT con el segundo escenario del 
estudio prospectivo se puede plantear la siguiente relación, las fortalezas (lenguaje, territorio, costumbres, cultura) que como pueblo conservan frente a las necesidades que como minoría marginada tienen, pueden conciliarse de manera positiva, es decir los Tsáchilas utilizan correctamente sus fortalezas para satisfacer sus necesidades sin perder su identidad y cohesión social, este escenario tiene una probabilidad de ocurrencia del $19.4 \%$. Por otro lado puede suceder que utilizan de una forma negativa sus fortalezas, lo que significa una pérdida de sus prácticas vivenciales (competencia) y recursos disponibles (mercantilización de la tierra) por satisfacer sus necesidades, esta realidad tiene una probabilidad de ocurrencia del 80,6\%.

Finalmente está el escenario favorable que coexistiría la situación en la cual los Tsáchilas logran una correcta convergencia entre satisfacción de las necesidades (desarrollo) y armonía comunitaria (Seke Sonano). Este futuro tiene una probabilidad de realizarse del $16.8 \%$ y supone compromiso colectivo y una disposición al intercambio étnico razonable (Tsáchilas-Mestizos).

\section{Conclusiones}

La participación de expertos, como agregados culturales de cuatro países vinculados al continente americano, asiático y africano, permitió examinar los componentes macro ambientales de la investigación, por otra parte el contacto directo con 207 miembros de la Nacionalidad Tsáchila en 7 comunas dispersas en 100.50 kilómetros cuadrados, catalizó un diagnóstico exhaustivo que fundamento la identificación de 43 variables y 
31 actores, que bajo un procesamiento lógico y secuencial de 3 software, se obtuvo la conformación de 64 escenarios posibles sujetos a una probabilidad relativa de ocurrencia, los cuales son confrontables con la visión general del Plan de Vida de la Etnia prevista para el año 2026.

La deliberación y juicio del grupo de expertos en la fase de reflexión colectiva, ha determinado 31 actores que mantienen vinculación con las 43 variables identificadas, y en función a los criterios de influencia y dependencia evidenciados en el histograma MMIDI del sistema MACTOR, se procede a validar que los stakeholders de mayor incidencia en la Nacionalidad Tsáchila, conciernen al Gobierno Central, la población mestiza que se encuentra adyacente a la zona geográfica donde se ubican las comunas, la Asamblea Nacional de la República y los Gobiernos Autónomos tanto el Municipal como el Provincial, por mantener la jurisdicción en ésta localización especifica del País.

Los Tsáchilas tienen dos propósitos fundamentales, por un lado buscan oportunidades que les permita alcanzar niveles de desarrollo y por otro intentan sobrevivir étnica y culturalmente, estos dos objetivos representan paradigmas de vida distintos por lo que se concluye que existe un antagonismo (cooperativismo vs Individualismo) que condicionan la realidad presente y futura de este pueblo.

\section{Referencias Bibliográficas}


Canelos H. (2004), El mestizaje de los Tsáchilas, Santo Domingo de los Colorados, Ecuador: Universitaria.

Cely, A. V. (1999). Metodología de los escenarios para estudios prospectivos. Ingeniería $\quad$ Investigación, 26-35. http://www.revistas.unal.edu.co/index.php/ingeinv/article/view/21296/22265

Gobierno de la Provincia de Santo Domingo de los Tsáchilas y Secretaría de Pueblos, Movimientos Sociales y Participación Ciudadana (2012), Plan de Vida de la Nacionalidad Tsa'chila, Santo Domingo de los Tsáchilas, Ecuador.

Godet, M. (1993). De la anticipación a la acción. Manual de Prospectiva y Estrategia. Barcelona.

Godet, M. (2004). Metodología de Investigación de Futuros. Recuperado el 2 de Marzo de 2015, de Análisis estructural, MICMAC y estrategia de los actores, MACTOR:

http://guajiros.udea.edu.co/fnsp/cvsp/politicaspublicas/godet_analisis_estructur al.pdf

Godet, M. (Abril de 2009).LIPSOR: La prospectiva estratégica para las empresas y los territorios. Obtenido de Serie de investigación $\mathrm{N}^{\mathrm{o}}$ 10: http://www.laprospective.fr/dyn/francais/actualites/SR10vSpa.pdf 
Godet M. \& Durance P. (2011), La prospectiva estratégica para las empresas y los territorios, Organización de las Naciones Unidas para la Educación, la Ciencia y la Cultura.

Moseley, Christopher (ed.). 2010. Atlas de las lenguas del mundo en peligro, 3ra edición. París, Ediciones UNESCO. Versión en línea: http://www.unesco.org/culture/en/endangeredlanguages/atlas

Senplades (2013), Plan Nacional del Buen vivir, Quito, Ecuador: Senplades

Vásquez, J. M., \& Ortegón, E. (2006). Manual de prospectiva y decisión estratégica: bases teóricas e instrumentos para América Latina y el Caribe (Vol. 51). United Nations Publications. http://archivo.cepal.org/pdfs/ 